\title{
Index of Instruments
}

Adaptive Steerable Imaging Array 167

AT Compact Array

Berkeley Illinois Maryland Association (BIMA)

$113,227,355,358$

Big Optical Array

113,235

Calar Alto $3.5 \mathrm{~m}$

151,177

CFHT

416

CHARON - III

273,391

188

China IR Optical Interferometry (CIROI)

171

COAST

163

CSO 10-m

235

Dodaira Observatory

297

ESO NTT

379

ESO VLT Interferometer

143,373

GHRIL on WHT

395

GI2T

155

Goldstone $70 \mathrm{~m}$

457

Gurushikar $1.2 \mathrm{~m}$ telescope

376

Hat Creek (BIMA) mm array

349

HIPPARCOS

424,443

HST

$61,401,404$

Infrared Spatial Interferometer (ISI)

$19,257,383$

IRAM

237

235

JCMT

287

327,333

299

$128,335,343$

193

352

$151,407,410,413,427,448$

167

364,379

236

101

113

297

485

236

442,481

27,237 
SCASIS

SHEVE array

Starfire Optical Range

SUSI

TDRSS - OVLBI

USA/European VLBI networks

USNO Astrometric Interferometer

Vacuum Tower Telescope

VLA

VLBA

VSOP

WHT $4 \mathrm{~m}$

WIRO $2.3 \mathrm{~m}$

WSRT
419

131

293

$135,181,184,224,302$

479

367

174,427

370,398

$113,212,243,335,401,457$

$105,113,117,125$

442,483

211,416

364

113 